\title{
Orographic effects on a 3-D barotropic lee wave across a meso-scale mountain corner
}

\author{
Prasanta Das $^{1} \cdot$ Somenath Dutta $^{2} \cdot$ Shyamal Kumar Mondal $^{3}$
}

Received: 7 May 2016/ Accepted: 4 June 2016/Published online: 9 July 2016

(C) Springer International Publishing Switzerland 2016

\begin{abstract}
An attempt has been made to study 3-D lee waves associated with an adiabatic, non-viscous, Bousisnesq, non-rotating, stationary dry mean flow across a mesoscale Mountain Corner hills (MCH). For simplicity, the far upstream undisturbed basic flow is assumed to have horizontal components of wind $(\mathrm{U}, \mathrm{V})$ and Brunt Vaisala frequency $(\mathrm{N})$ to be independent of height. $\mathrm{MCH}$ is synthesized by the intersection of two semi-infinite ridges, parallel to the horizontal axes of co-ordinates. The primitive equations are used in $\mathrm{z}$-coordinate. The nonlinear governing equations are linearized by using perturbation technique. The linearized equations are then subjected to two-dimensional Fourier transform. The 2nd order ordinary differential equation in the Fourier transform $\hat{w}$ of perturbation vertical velocity $w^{\prime}$ is obtained by some algebraic simplification. This equation has been solved by using the radiational upper boundary condition and the lower boundary condition imposed by the profile of the hill at surface. The vertical velocity $\left(w^{\prime}\right)$ is represented by double integral, which is very complicated. This double integral is evaluated by using asymptotic technique.
\end{abstract}

Prasanta Das

pdas.math1986@gmail.com

Somenath Dutta

dutta.drsomenath@gmail.com

Shyamal Kumar Mondal

shyamal_260180@yahoo.com

1 Department of Mathematics, Ramananda College, Bishnupur, Bankura 722122, West Bengal, India

2 India Meteorological Department (IMD), Pune 411008, India

3 Department of Applied Mathematics with Oceanology and Computer Programming, Vidyasagar University, Midnapore 721102, West Bengal, India
Keywords Mountain Corner hills · Lee wave · Orographic effects

\section{Introduction}

Weather and climate of a place is substantially influenced by the presence of orographic barriers at that place. Influence of orographic barriers on airflow depends on the scale of the barrier as well as scale of flow.

When a stably stratified air parcel flows an orographic barrier, gravity waves are excited to propagate upward direction under certain conditions of thermal stability and airflow stratification. It is also known as internal gravity waves (IGW). The IGW can propagate horizontally and vertically to a great distance carrying energy and momentum to higher levels in the atmosphere. Sometimes, they are associated with the formation of clear air turbulence (CAT). The information about standing waves, which under favorable meteorological conditions form on the lee side of the mountain barrier, is very important for the safety of aviation. Many aircraft accidents reported in mountainous areas are often attributed to the vertical velocities of large magnitude associated with the lee waves. Hence the studies on lee waves associated with air flow across an orographic barrier have an important bearing on the safety of aviation.

Theoretical investigation in this field can broadly be divided into two types. In one type, the orographic barrier has been assumed to have an infinite extension in the cross mean flow direction, which constrains the perturbation to be 2-dimensional. Such studies may be traced back to the works of Lyra (1943), Queney (1947, 1948), Scorer (1949), Sawyer (1960), Sarker (1965, 1966, 1967), De (1973), Sinha Ray (1988), Kumar et al. (1998), etc. In another type 
of theoretical studies, the orographic barrier has been assumed to have finite extension in both directions, viz., along and across the basic flow, due to which the perturbations are essentially of 3-dimensional. Such studies may be traced back to the work of Scorer and Wilkinson (1956), Wurtele (1957), Crapper (1959), Sawyar (1962), Das (1964), Onishi (1969), Smith (1979, 1980), Dutta et al. (2002), Dutta (2003, 2005), Kumar (2007), Das et al. (2013, 2016), etc.

In some of the above studies, wind and stability were assumed to be either invariant with height or assumed to have some analytical behavior with height. Solutions for such studies were essentially obtained by analytical method. In other studies, realistic vertical variation of wind and stability were considered and the solution obtained using quasi numerical or numerical method.

In India studies on the effect of an orographic barrier on airflow have been addressed by Das (1964), Sarker (1965, 1966, 1967), Sarker et al. (1978), De (1971, 1973), Sinha Ray (1988), Dutta et al. (2002), Dutta (2005), Dutta et al. (2006), Kumar et al. (2005), Das et al. (2016), etc. In all the studies the barrier (2-D) or the major ridge axis (3D) of the barrier has been assumed to be extended broadly either in the East-West (EW) direction or in North-South (NS) direction.

In India in the northeast region, the Khasi-Jayantia (KJ) hill is broadly EW oriented whereas the Assam-Burma (AB) hill is broadly NS oriented and they meet at almost right angle forming a mountain corner to the northeast. It is believed that weather and climate in that region are neither controlled by $\mathrm{KJ}$ hill alone nor it is controlled by $\mathrm{AB}$ hill alone, rather they are controlled by their combined effect. To address the problem of this combined effect, one has to investigate the effect of the above mountain corner on airflow and rainfall in that region.

From the foregoing discussion it appears that the corner mountain wave problem has not been addressed so far. The objective of the present study is to develop a 3-D meso scale lee wave model for air flow across an idealized mountain corner and apply it for the corner effect of $\mathrm{KJ}$ hills and AB hills.

\section{Database}

Guwahati $\left(26.19^{\circ} \mathrm{N}\right.$ latitude and $91.73^{\circ} \mathrm{E}$ longitude) is the only radio sonde station to the upstream of both $\mathrm{KJ}$ hills and $\mathrm{AB}$ hills. Accordingly for the present study we have used the RS/RW data of Guahati for those dates, which corresponds to the observed lee waves across $\mathrm{ABH}$, as reported by De $(1970,1971)$ and Farooqui and De (1974) and has been obtained from Archive of India Meteorological Department, Pune.

\section{Methodology}

In this paper, we shall develop a three dimensional mesoscale lee wave model considering the basic flow consist of both zonal component (U) and meridional component (V). To develop this model we have considered the flow is an adiabatic, steady state, non-rotating, non-viscous, Bousisnesq. Also we assume two components $(\mathrm{U}, \mathrm{V})$ of basic flow and Brunt-vaisala frequency $(\mathrm{N})$ are invariant with height. Under these assumptions the linearized governing equations are:

$U \frac{\partial u^{\prime}}{\partial x}+V \frac{\partial u^{\prime}}{\partial x}=-\frac{1}{\rho_{0}} \frac{\partial p^{\prime}}{\partial x}$

$U \frac{\partial v^{\prime}}{\partial x}+V \frac{\partial v^{\prime}}{\partial x}=-\frac{1}{\rho_{0}} \frac{\partial p^{\prime}}{\partial y}$

$U \frac{\partial w^{\prime}}{\partial x}+V \frac{\partial w^{\prime}}{\partial x}=-\frac{1}{\rho_{0}} \frac{\partial p^{\prime}}{\partial z}+g \frac{\theta^{\prime}}{\theta_{0}}$

$\frac{\partial u^{\prime}}{\partial x}+\frac{\partial v^{\prime}}{\partial y}+\frac{\partial w^{\prime}}{\partial z}=0$

$U \frac{\partial \theta^{\prime}}{\partial x}+V \frac{\partial \theta^{\prime}}{\partial y}+w^{\prime} \frac{d \theta_{0}}{d z}=0$

where $U, V, \rho_{0}, \theta_{0}$ are, respectively, zonal state wind, meridional wind, density and potential temperature and $u^{\prime}, v^{\prime}, w^{\prime}, p^{\prime}, \rho^{\prime}, \theta^{\prime}$ are, respectively, the perturbation zonal wind, meridional wind, vertical wind, pressure, density, and potential temperature. Since the perturbation quantities, $u^{\prime}, v^{\prime}, w^{\prime}, p^{\prime}, \rho^{\prime}, \theta^{\prime}$, etc., are all continuous functions of $x, y, z$ hence their horizontal variation may be represented by a double Fourier integral, such as,

$u^{\prime}(x, y, z)=\frac{1}{4 \pi^{2}} \int_{-\infty}^{\infty} \int_{-\infty}^{\infty} \hat{u}(k, l, z) e^{i(k x+l y)} \mathrm{d} k \mathrm{~d} l$

where $\hat{u}(k, l, z)=\int_{-\infty}^{\infty} \int_{-\infty}^{\infty} u^{\prime}(x, y, z) e^{-i(k x+l y)} \mathrm{d} x \mathrm{~d} y$ is the double Fourier transform of $u^{\prime}(x, y, z)$.

Perform double Fourier transform to (1)-(5) we get

$i(k U+l V) \hat{u}=-i k \frac{\hat{p}}{\rho_{0}}$

$i(k U+l V) \hat{v}=-i l \frac{\hat{p}}{\rho_{0}}$

$i(k U+l V) \hat{w}=-\frac{1}{\rho_{0}} \frac{\partial \hat{p}}{\partial z}+g \frac{\hat{\theta}}{\theta_{0}}$

$i k \hat{u}+i l \hat{v}+\frac{\partial \hat{w}}{\partial z}=0$

$i(k U+l V) \hat{\theta}+\hat{w} \frac{\mathrm{d} \theta_{0}}{\mathrm{~d} z}=0$ 
Eliminating $\hat{u}, \hat{v}$ from the Eqs. (6)-(10) we get

$\frac{\partial^{2} \hat{w}}{\partial z^{2}}+\frac{1}{\rho_{0}} \frac{\mathrm{d} \rho_{0}}{\mathrm{~d} z} \frac{\partial \hat{w}}{\partial z}+\left(k^{2}+l^{2}\right)\left[\frac{N^{2}}{(k U+l V)^{2}}-1\right] \hat{w}=0$

where, $N=\sqrt{\frac{g}{\theta_{0}} \frac{\mathrm{d} \theta_{0}}{\mathrm{~d} z}}$ is the Brunt-Vaisala frequency.

Now, putting $\hat{w}(k, l, z)=\sqrt{\frac{\rho_{0}(o)}{\rho_{0}(z)}} \hat{w}_{1}(k, l, z)$ in Eq. (11), we get

$\frac{\partial^{2} \hat{w}_{1}}{\partial z^{2}}+\left[\frac{N^{2}}{(k U+l V)^{2}}-1\right]\left(k^{2}+l^{2}\right) \hat{w}_{1}=0$

$\frac{\partial^{2} \hat{w}_{1}}{\partial z^{2}}+m^{2} \hat{w}_{1}=0$

where,

$m^{2}=\left[\frac{N^{2}}{(k U+l V)^{2}}-1\right]\left(k^{2}+l^{2}\right)$

For vertically propagating wave, the solution of (12) can be taken as

$\hat{w}_{1}(k, l, z)=\hat{w}_{1}(k, l, 0) e^{i m z}$

Now, at the surface the airflow follows the contour of the mountain, the profile is given by

$h(x, y)=\frac{H}{2}\left[\frac{a^{2}}{a^{2}+\left(x-x_{0}\right)^{2}}+\frac{b^{2}}{b^{2}+\left(y-y_{0}\right)^{2}}\right]$

Where ' $\mathrm{H}$ ' be height of $\mathrm{MCH}$ and the profile of (15) is given by Fig. 1. The Gray and Sky color of this figure is the Corner Montain which we are interested to study.

Now the double Fourier transform of (15) is given by

$\hat{h}(k, l)=-\frac{i H}{2}\left[\frac{a}{l} e^{-a k-k i x_{0}}+\frac{b}{k} e^{-b l-l i y_{0}}\right]$

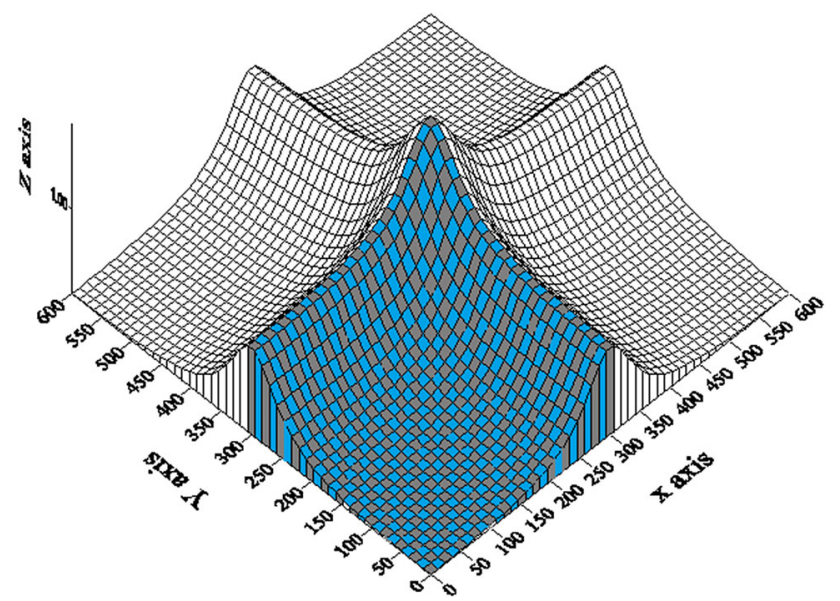

Fig. 1 Profile of Mountain Corner hills
Thus at the ground surface (at $z=0$ ) the streamline displacement $\eta^{\prime}(x, y, z=0)$ follows the ground contour

$$
\begin{aligned}
\eta^{\prime}(x, y, z=0) & =\frac{H}{2}\left[\frac{a^{2}}{a^{2}+\left(x-x_{0}\right)^{2}}+\frac{b^{2}}{b^{2}+\left(y-y_{0}\right)^{2}}\right] \\
& =h(x, y)
\end{aligned}
$$

Hence

$\hat{\eta}(k, l, 0)=\hat{h}(k, l)=-\frac{i H}{2}\left[\frac{a}{l} e^{-a k-i k x_{0}}+\frac{b}{k} e^{-b l-i l y_{0}}\right]$

Now the linearized lower boundary condition for $w_{1}^{\prime}$ is given by

$w_{1}^{\prime}(x, y, 0)=w^{\prime}(x, y, 0)=U \frac{\partial \eta^{\prime}(x, y, 0)}{\partial x}+V \frac{\partial \eta^{\prime}(x, y, 0)}{\partial y}$

Hence

$\hat{w}_{1}(k, l, 0)=i(k U+l V) \hat{\eta}(k, l, 0)$

Then from (14) and (18) we get

$\hat{w}_{1}(k, l, z)=i(k U+l V) \hat{\eta}(k, l, 0) e^{i m z}$

Hence

$w_{1}^{\prime}(x, y, z)=\int_{0}^{\infty} \int_{0}^{\infty} \hat{w}_{1}(k, l, z) e^{i(k x+l y)} \mathrm{d} k \mathrm{~d} l$

Therefore

$$
\begin{aligned}
w^{\prime}(x, y, z)= & \frac{H}{8 \pi^{2}} \sqrt{\frac{\rho_{0}(o)}{\rho_{0}(z)}} R e \int_{0}^{\infty} \int_{0}^{\infty}(k U+l V) \\
& \times\left[\frac{a}{l} e^{-a k-i k x_{0}}+\frac{b}{k} e^{-b l-i l y_{0}}\right] e^{i(k x+l y+m z)} \mathrm{d} k \mathrm{~d} l
\end{aligned}
$$

To evaluate (21) we make following substation: $k=\frac{\lambda N}{\sqrt{U^{2}+V^{2}} \cos \alpha}, l=\frac{\mu N}{\sqrt{U^{2}+V^{2}} \cos \alpha}$ and $x=X \frac{\sqrt{U^{2}+V^{2}} \cos \alpha}{N}$, $y=Y \frac{\sqrt{U^{2}+V^{2}} \cos \alpha}{N}, z=Z \frac{\sqrt{U^{2}+V^{2}} \cos \alpha}{N}$ where $\alpha$ be the angle between the basic flow vector $\vec{V}=U \vec{i}+V \vec{j}$ and the wave number vector $\vec{K}=k \vec{i}+l \vec{j}$.

Now

$\vec{V} \cdot \vec{K}=|\vec{V}||\vec{K}| \cos \alpha$

Now

$$
\begin{aligned}
& m^{2}=\left[\frac{N^{2}}{(k U+l V)^{2}}-1\right]\left(k^{2}+l^{2}\right) \\
& =\left[\frac{N^{2}}{\left(U^{2}+V^{2}\right) \cos ^{2} \alpha}-\frac{\left(\lambda^{2}+\mu^{2}\right) N^{2}}{\left(U^{2}+V^{2}\right) \cos ^{2} \alpha}\right] \\
& =\frac{N^{2}}{\left(U^{2}+V^{2}\right) \cos ^{2} \alpha}\left[1-\lambda^{2}-\mu^{2}\right]
\end{aligned}
$$



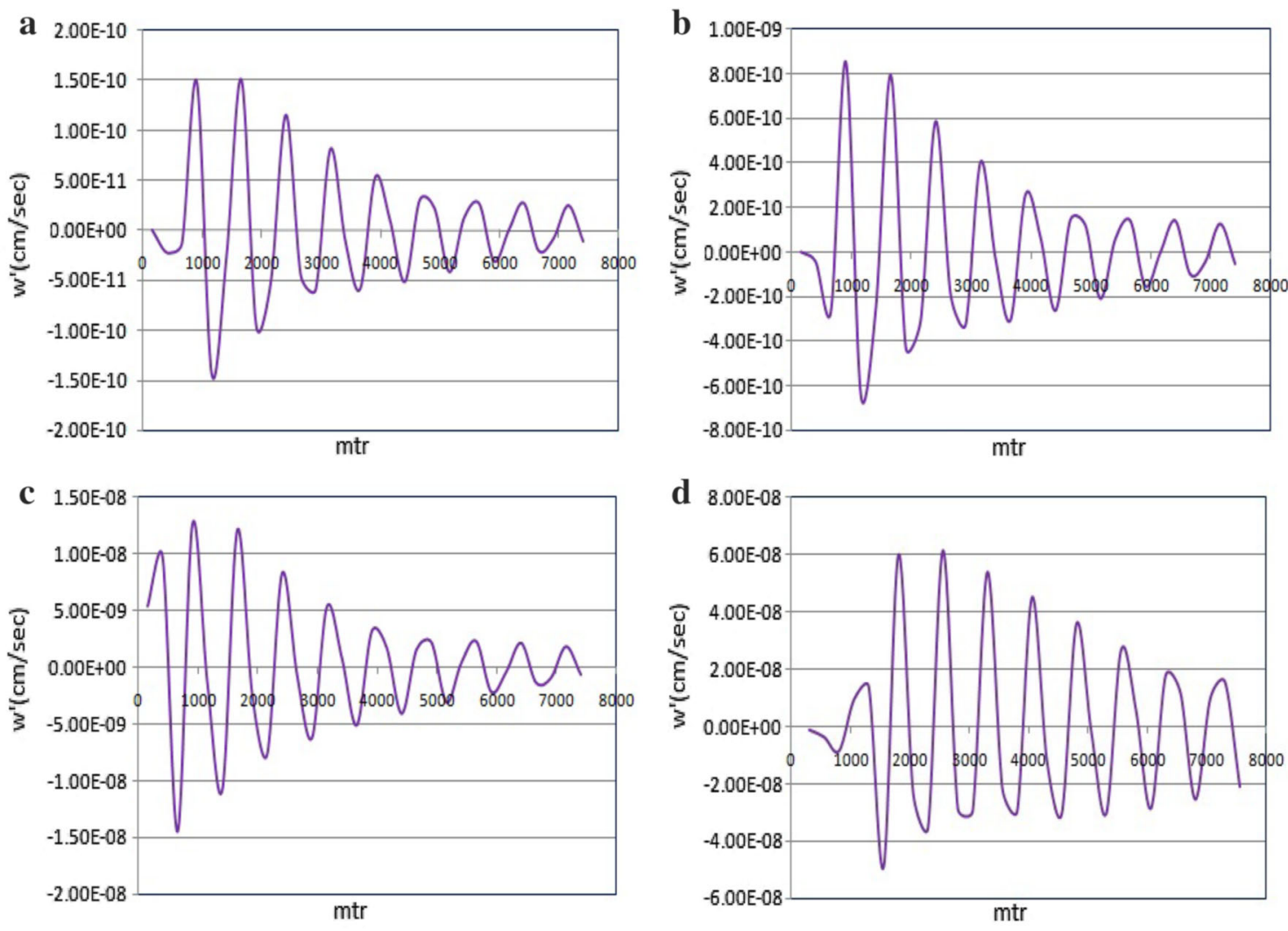

Fig. 2 a-d Downstream variation of $w^{\prime}$ along the line ' $U y-V x=-50$ ' at the levels $1.5,3,6$ and $10 \mathrm{~km}$, respectively

Therefore,

$m=\frac{N}{\sqrt{\left(U^{2}+V^{2}\right)} \cos \alpha} \sqrt{1-\lambda^{2}-\mu^{2}}$

Using the above substitutions, the integral in (21) has been approximated using asymptotic method, following Hsu (1948).

$$
\begin{aligned}
& w^{\prime}(x, y, z)= \exp \left(\frac{g-R^{*} \gamma}{2 R^{*} T} z\right) \\
& \times \frac{N H Z r_{1}}{16 R_{1}^{2}}\left[\begin{array}{c}
\frac{a}{y} \exp \left(\frac{-a x N r_{1}}{R_{1} R_{4}}\right) \cos \left(\frac{N R_{1} r_{1}}{R_{4}}-\frac{N x x_{0} r_{1}}{R_{1} R_{4}}\right)+ \\
\frac{b}{x} \exp \left(\frac{-b y N r_{1}}{R_{1} R_{4}}\right) \cos \left(\frac{N R_{1} r_{1}}{R_{4}}-\frac{N y y_{0} r_{1}}{R_{1} R_{4}}\right)
\end{array}\right]
\end{aligned}
$$

Details of the above derivation is given in "Appendix", where $\quad r_{1}=\sqrt{x^{2}+y^{2}}, \quad R_{1}=\sqrt{x^{2}+y^{2}+z^{2}} \quad$ and $R_{4}=U x+V y$

\section{Result and discussion}

Now the Eq. (24), it is clearly shown that at the ground surface (i.e., $\mathrm{z}=0$ ) $w^{\prime}$ vanishes. This should be so for lee wave (Dutta et al. 2002). Using the Eq. (24), the perturbation vertical velocity $\left(w^{\prime}\right)$ is computed at different levels for the typical lee wave case across the corner mountain hill, the order of magnitude ' $U$ ', ' $\mathrm{V}$ ' and ' $\mathrm{N}$ ' are $1 \mathrm{~m} / \mathrm{sec}, 1 \mathrm{~m} / \mathrm{sec}$ and $0.01 / \mathrm{s}$, respectively. We computed $w^{\prime}$ at four levels only, viz., at 1.5, 3, 6 and $10 \mathrm{~km}$ above mean see level, which approximately resemble to $850,700,500$ and $300 \mathrm{hPa}$, respectively. The downstream variation of $w^{\prime}$ along the line ' $U y-V x=-50$ ' at the levels $1.5,3,6$ and $10 \mathrm{~km}$ above the mean see level are shown in the Fig. $2 \mathrm{a}-\mathrm{d}$. From these figures we see that the downstream decay at above mentioned levels in the amplitude of $w^{\prime}$ about the line ' $U y-V x=-50$ '.

The contours of perturbation vertical velocity $\left(w^{\prime}\right)$ at the levels $1.5,3,6$ and $10 \mathrm{~km}$ above the mean see level, which approximately resemble to $850,700,500$ and $300 \mathrm{hPa}$, respectively, are shown in Fig. 3a-d. These figures show that the maximum updraft regions are 'crescent shaped' and symmetric about the line line ' $U y-V x=0$ '. Wurtele (1957) showed crescent shaped updraft region, symmetric about $\mathrm{x}$-axis, taking constant basic flow, with only component (only $\mathrm{U}$ component) across the barrier. Dutta et al. (2002) and Das et al. (2013) they obtained horseshoes shaped updraft region symmetric about the line ' $y=0$ ' and taking constant basic flow $(U)$ with only $\mathrm{U}$-component. In the present study, due to the presence of $\mathrm{V}$-component, there is a meridional forcing acting at all level, causing the 

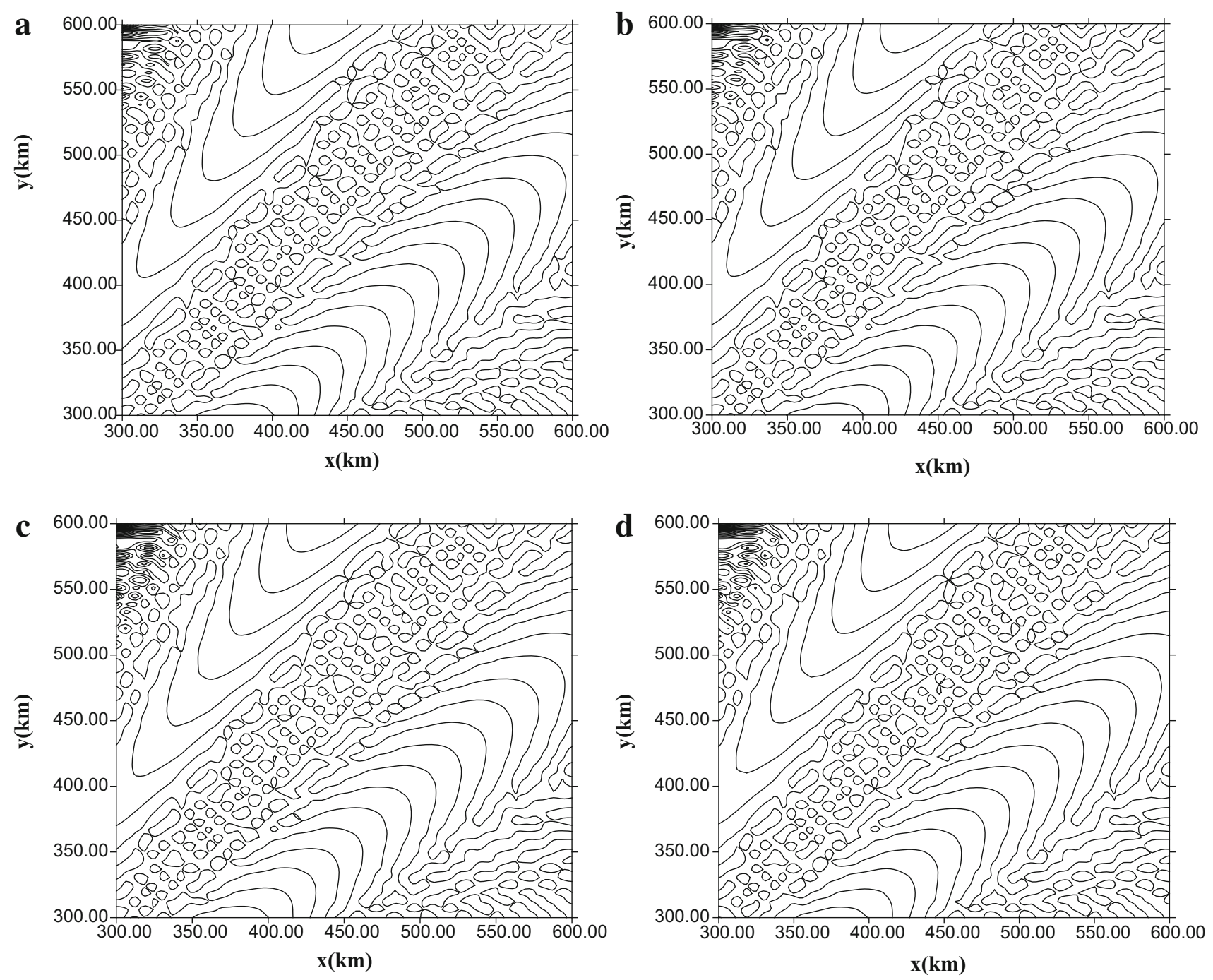

Fig. 3 a-d Contours of $w^{\prime}$ across the corner mountain barrier at the levels 1.5, 3, 6 and $10 \mathrm{~km}$, respectively

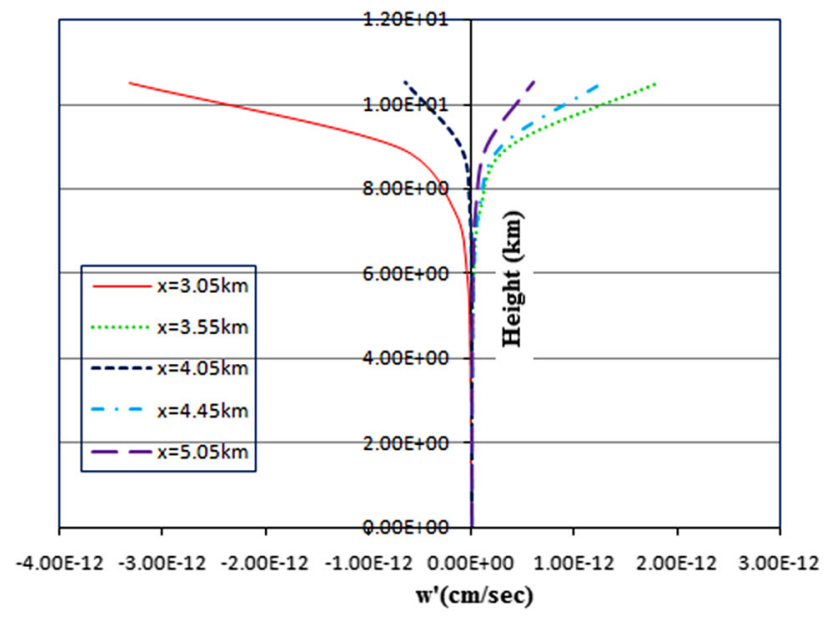

Fig. 4 Vertical profile of $w^{\prime}$ along the line ' $U y-V x=-50$ ' at different heights and at different downstream locations symmetric(about the line $\mathrm{y}=0$ ) crescent shaped updraft region to rotate through an angle $\tan ^{-1}\left(\frac{V}{U}\right)$.

The vertical profile of perturbation vertical velocity $\left(w^{\prime}\right)$ along the line ' $U y-V x=-50$ ' at different heights, at different downstream locations is shown in Fig. 4. It is seen that the perturbation vertical velocity $\left(w^{\prime}\right)$ at every point of downstream location does not form alternatively positive and negative, i.e., cellular structure.

\section{Conclusions}

In this investigation, we have presented the orographic effects on a 3-D barotropic lee wave across a meso-scale mountain corner following the asymptotic approach. In the sequel, we have made some interesting observation. Moreover, 
1. The asymptotic solution for perturbation vertical velocity $\left(w^{\prime}\right)$, along the line $U y-V x=-50$, the downstream decay at the mentioned levels.

2. The contours of the perturbation vertical velocity $\left(w^{\prime}\right)$ at the above mentioned levels are symmetric about the central plane along the line $U y-V x=0$ and maximum updraft regions are crescent shaped which are inclined at an angle $\tan ^{-1}\left(\frac{V}{U}\right)$.

3. The vertical profile of $w^{\prime}$ along the line $U y-V x=-50$, is obtained by asymptotic method and does not form the cellular structure.

Acknowledgments Authors are grateful to Dr. U.S. De, Former Addl. Director General of Meteorology of India Meteorological Department and to Dr. G. Krishnakumar, Scientist-E, Head, National Data Centre, India Meteorological Department, PUNE 411005, Maharashtra, India and to Prof. H.P. Mazumdar, Scientist, Physics and Applied Mathematics Unit, ISI, Kolkata, India and to Prof. M. Maiti, Retd Professor, Dept. of Applied Mathematics, Vidyasagar University, Midnapore, West Bengal, India for their kind valuable suggestions and guidance.

\section{Appendix}

Hsu's (1948) theorem: In the theorem, he has shown that if $\Phi(x, y), h(x . y)$ and $f(x, y)=e^{h(x, y)}$ are continuous functions defined on a region $\mathrm{S}$ such that,

(1) $\Phi(x, y),[f(x, y)]^{n}$ are absolutely integrable over $\mathrm{S}$ for $\mathrm{n}=0,1,2$.

(2) $\frac{\partial f}{\partial x}, \frac{\partial^{2} f}{\partial x^{2}}, \frac{\partial f}{\partial y}, \frac{\partial^{2} f}{\partial y^{2}}$ exist and continuous over S.

(3) $h(x, y)$ has an absolute maximum value at an interior pt $\left(x_{0}, y_{0}\right)$ such that

At $\left(x_{0}, y_{0}\right) \frac{\partial h}{\partial x}=\frac{\partial h}{\partial y}=0, \frac{\partial^{2} h}{\partial x^{2}} \frac{\partial^{2} h}{\partial y^{2}}-\left(\frac{\partial^{2} h}{\partial x \partial y}\right)^{2}>0$

(4) $\Phi(x, y)$ is continuous at $\left(x_{0}, y_{0}\right)$ and $\Phi\left(x_{0}, y_{0}\right) \neq 0$. Now if $\mathrm{C}$ be an analytic curve passing through the point $\left(x_{0}, y_{0}\right)$, such that the region $\mathrm{S}$ is divided into two sub regions $S_{1}$ and $S_{2}$. Then the integral

(5) $\iint \Phi(x, y)[f(x, y)]^{n} d s$ taken over either of $S_{1}$ and $S_{2}$ asymptotic to

$$
\frac{\pi \Phi\left(x_{0}, y_{0}\right)\left[f\left(x_{0}, y_{0}\right)\right]^{n}}{\sqrt[n]{\left[\frac{\partial^{2} h \partial^{2} h}{\partial x^{2}} \partial y^{2}-\left(\frac{\partial^{2} h}{\partial x \partial y}\right)^{2}\right]_{\left(x_{0}, y_{0}\right)}}}
$$

Using $\sqrt{\frac{\rho_{0}(0)}{\rho_{0}(z)}} \approx \exp \left(\frac{g-R^{*} \gamma}{2 R^{*} T} z\right)$ (Dutta 2003) in the integral (21), has been transformed to $\frac{H}{8 \pi^{2}} \exp \left[\frac{z\left(g-R^{*} \gamma\right)}{2 R^{*} T}\right] I_{1}$, where, $R^{*}$ is the gas constant and $\gamma$ is the ratio of two specific heat of gas.

$$
\begin{aligned}
I_{1}= & \iint_{0}^{\infty}(k U+l V)\left[\frac{a}{l} e^{-\left(a+i x_{0}\right) k}+\frac{b}{k} e^{-\left(b+i y_{0}\right) l}\right] \\
& \times \exp [i(k x+l y+m z)] d k d l \\
= & \iint_{0}^{\infty} p(\lambda U+\mu V)\left[\frac{a}{\mu p} e^{-\left(a+i x_{0}\right) \lambda p}+\frac{b}{\lambda p} e^{-\left(b+i y_{0}\right) \mu p}\right] \\
& \times \exp [i(\lambda X+\mu Y+v Z)] p^{2} d \lambda d \mu=I_{11}+I_{12}
\end{aligned}
$$

Where,

$$
\begin{aligned}
& I_{11}=\iint_{0}^{\infty}(\lambda U+\mu V)\left[\frac{a}{\mu} e^{-\left(a+i x_{0}\right) \lambda p} \exp [i(\lambda X+\mu Y+v Z)]\right] p^{2} d \lambda d \mu \\
& I_{12}=\iint_{0}^{\infty}(\lambda U+\mu V)\left[\frac{b}{\lambda} e^{-\left(b+i y_{0}\right) \mu p} \exp \left[i\left(\lambda X_{1}+\mu Y_{1}+v Z_{1}\right)\right]\right] p^{2} d \lambda d \mu
\end{aligned}
$$

Now to evaluate the double integrals $I_{11}$ and $I_{12}$ following substitutes are made:

$\lambda=\tau \cos \beta, \mu=\tau \sin \beta, X=R \cos \theta, Y=R \operatorname{Sin} \theta$.

Therefore, $\lambda X+\mu Y+v Z=\tau R \cos (\beta-\theta)+Z \sqrt{1-\tau^{2}}=\sigma$ (say)

Hence,

$I_{11}=\int_{0}^{\infty} \int_{-\pi / 2}^{\pi / 2} \frac{\tau a p^{2}(U \cos \beta+V \sin \beta)}{\sin \beta} e^{-a \tau p \cos \beta-i x_{0} p \tau \cos \beta} e^{i \sigma} d \tau d \beta . \quad$ This double integral is difficult to evaluate analytically. So they are amenable to the method of stationary phase, following Hsu's (1948). According to this method, first those points $(\tau, \beta)$ in the wave number domain are found out, where the phase $\sigma$ is stationary. Those points are termed as saddle points. Then the entire integrand is expanded in Taylor's series about the saddle point and the first term of the expansion is retained as the asymptotic approximation of the integrals, which is valid at far down wind location of the mountain. Details of the theorem, given by Hsu's (1948). Following Hsu's (1948),

Let $\phi(\tau, \beta)=\frac{\tau a p^{2}(U \cos \beta+V \sin \beta)}{\sin \beta} e^{-a \tau p \cos \beta-i x_{0} p \tau \cos \beta} \quad$ and $h(\tau, \beta)=i \tau R \cos (\beta-\theta)+Z_{1} \sqrt{1-\tau^{2}}$ and $f(\tau, \beta)=e^{h(\tau, \beta)}$. $\frac{\partial h}{\partial \tau}=0$ and $\frac{\partial h}{\partial \tau}=0$ Gives the stationary point $\left(\tau_{0}, \beta_{0}\right)$ as

$$
\begin{aligned}
\tau_{0} & =\frac{R_{1}}{\sqrt{Z^{2}+R_{1}^{2}}}=\sqrt{\frac{X^{2}+Y^{2}}{X^{2}+Y^{2}+Z^{2}}}=\sqrt{\frac{x^{2}+y^{2}}{x^{2}+y^{2}+z^{2}}} \\
& =\frac{r_{1}}{R_{1}} \text { and } \beta_{0}=\theta,
\end{aligned}
$$

Where $r_{1}^{2}=x^{2}+y^{2}$ and $R_{1}^{2}=x^{2}+y^{2}+z^{2}$. It can be shown that at stationary point $\left(\tau_{0}, \beta_{0}\right)$.

$$
p=\frac{N r_{1}}{R_{4}}, \sigma=\frac{N r_{1} R_{1}}{R_{4}}, \tau p \cos \beta=\frac{N x r_{1}}{R_{1} R_{4}}, \frac{U \cos \beta+V \sin \beta}{\sin \beta}=\frac{R_{4}}{y},
$$

Where $R_{4}=U x+V y$. 


$$
\begin{gathered}
\begin{aligned}
\phi\left(\tau_{0}, \beta_{0}\right) & =\frac{\tau a p^{2}(U \cos \beta+V \sin \beta)}{\sin \beta} e^{-a \tau p \cos \beta-i x_{0} p \tau \cos \beta} \\
& =\frac{N^{2} r_{1}^{3}}{R_{1} R_{4}} \frac{a}{y} \exp \left(\frac{-\mathrm{aNr}_{1} x}{\mathrm{R}_{1} \mathrm{R}_{4}}\right) \exp \left(\frac{-\mathrm{ix}_{0} \mathrm{Nr}_{1} x}{\mathrm{R}_{1} \mathrm{R}_{4}}\right)
\end{aligned} \\
\text { Now }\left(\frac{\partial^{2} h}{\partial \tau^{2}}\right)_{\left(\tau_{0}, \beta_{0}\right)}=-\frac{Z_{1}}{\left(1-\tau^{2}\right)^{\frac{3}{2}}}, \quad\left(\frac{\partial^{2} h}{\partial \tau^{2}}\right)_{\left(\tau_{0}, \beta_{0}\right)}=-\frac{Z_{1}}{\left(1-\tau^{2}\right)^{\frac{3}{2}}}, \\
\left(\frac{\partial^{2} h}{\partial \beta^{2}}\right)_{\left(\tau_{0}, \beta_{0}\right)}=-\tau R_{1} \cos (\beta-\theta) \quad \text { and } \quad\left(\frac{\partial^{2} h}{\partial \tau \partial \beta}\right)_{\left(\tau_{0}, \beta_{0}\right)}=-R_{1}
\end{gathered}
$$
$\sin (\beta-\theta)$.

Hence, $\quad\left[\left(\frac{\partial^{2} h}{\partial \tau^{2}}\right)\left(\frac{\partial^{2} h}{\partial \beta^{2}}\right)-\left(\frac{\partial^{2} h}{\partial \tau^{2}}\right)^{2}\right]_{\left(\tau_{0}, \beta_{0}\right)}=\frac{\tau_{0} R_{1} Z_{1}}{\left(1-\tau^{2}\right)^{\frac{3}{2}}}=$ $\frac{R^{2}\left(R^{2}+Z_{1}^{2}\right)}{Z_{1}^{2}}=\frac{N r_{1}^{2} R_{1}}{R_{4} z}>0$. Let us consider a path ' $\mathrm{C}$ ', in the region passing through the stationary point $\left(\tau_{0}, \beta_{0}\right)$,which has divided the region into two parts, viz., the upstream side and downstream side of the mountain. Hence following, Hsu (1948), the contribution of the integral $I_{11}$ to the downstream side can be approximated as

$$
\begin{aligned}
\frac{\pi \varphi\left(\tau_{0}, \beta_{0}\right)\left[f\left(\tau_{0}, \beta_{0}\right)\right]}{2 \sqrt{\left[\frac{\partial^{2} h \partial^{2} h}{\partial \tau^{2}} \frac{\partial^{2} \beta^{2}}{}-\left(\frac{\partial^{2} h}{\partial \tau \partial \beta}\right)^{2}\right]_{\left(\tau_{0}, \beta_{0}\right)}}}= & \frac{\pi}{2} \frac{N r_{1} z}{R_{1}^{2}} \frac{a}{y} \exp \left(\frac{-a N r_{1} x}{R_{1} R_{4}}\right) \\
& \times \exp \left(i\left[\frac{N r_{1} R_{1}}{R_{4}}-\frac{N r_{1} x_{0} x}{R_{1} R_{4}}\right]\right)
\end{aligned}
$$

Following similar argument, it can be seen that the contribution of the integral $I_{12}$ to the downstream side can be approximated as $\frac{\pi}{2} \frac{N r_{1} z}{R_{1}^{2}} \frac{b}{x} \exp \left(\frac{-b N r_{1} y}{R_{1} R_{4}}\right) \exp$ $\left(i\left[\frac{N r_{1} R_{1}}{R_{4}}-\frac{N r_{1} y_{0} y}{R_{1} R_{4}}\right]\right)$.

Hence

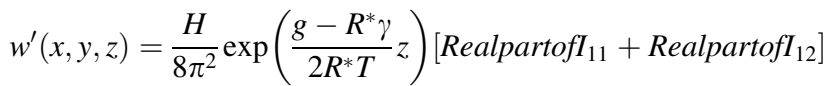

$$
\begin{aligned}
& w^{\prime}(x, y, z)=\exp \left(\frac{g-R^{*} \gamma}{2 R^{*} T} z\right) \frac{N H r_{1} z}{16 R_{1}^{2}} \\
& \times\left[\begin{array}{c}
\frac{a}{y} \exp \left(\frac{-a N r_{1} x}{R_{1} R_{4}}\right) \cos \left(\frac{N r_{1} R_{1}}{R_{4}}-\frac{N r_{1} x_{0} x}{R_{1} R_{4}}\right)+ \\
\frac{b}{x} \exp \left(\frac{-b N r_{1} y}{R_{1} R_{4}}\right) \cos \left(\frac{N r_{1} R_{1}}{R_{4}}-\frac{N r_{1} y_{0} y}{R_{1} R_{4}}\right)
\end{array}\right]
\end{aligned}
$$

\section{References}

Crapper GD (1959) A three dimensional study for waves in the lee of mountains. J Fluid Mech 6:51-76

Das PK (1964) Lee waves associated with a large circular mountain. Indian J Meteorol Geophys 15:547-554

Das P, Mondal SK, Dutta S (2013) Asymptotic solution for 3D lee waves across Assam-Burma hills. MAUSAM 64(3):501-516

Das P, Dutta S, Mondal SK, Khan A (2016) Momentum flux and energy flux associated with internal gravity waves excited by the Assam-Burma hills in India. Model Earth Syst Environ 2(2): $1-9$

De US (1970) Lee waves as evidenced by satellite cloud pictures. IJMG 21(4):637-642

De US (1971) Mountain waves over northeast India and neighbouring regions. Indian J Meteorol Geophys 22:361-364

De US (1973) Some studies on mountain waves. PhD thesis, Banaras Hindu University, India

Dutta S (2003) Some studies on the effect of orographic barrier on airflow. $\mathrm{PhD}$ thesis, Vidyasagar University, Midnapur, India

Dutta S (2005) Effect of static stability on the pattern of 3-D baroclinic lee wave across a meso-scale elliptical barrier. Meteorol Atmos Phys 90:139-152

Dutta S, Maiti M, De US (2002) Waves to the lee of a meso-scale elliptic orographic barrier. Meteorol Atmos Phys 81:219-235

Dutta S, Mukherjee AK, Singh AK (2006) Effect of Palghat gap on the rainfall pattern to the north and south of its axis. Mausam 57(4):675-700

Farooqui SMT, De US (1974) A numerical study of the mountain wave problem. Pure Appl Geophys 112:289-300

Hsu LC (1948) Approximation to a class of double integrals of functions of large numbers. Am J Math 70:698-708

Kumar N (2007) An analytical model of mountain wave for wind with shear. Math Comput Appl 12(1):11

Kumar P, Singh MP, Padmanabhan N, Natarajan N (1998) An analytical model for mountain waves in stratified atmosphere. Mausam 49(4):433-438

Kumar N, Roy Bhowmik SK, Ahmad N, Hatwar HR (2005) A mathematical model for rotating stratified airflow across KhasiJayantia hills. Mausam 56(4):771-778

Lyra G (1943) Theorie der stationaren Leewellenstromung in freier Atmosphare. Z Angew Math Mech 23:1-28

Onishi G (1969) A numerical method for three dimensional mountain waves. J Meteorol Soc Jpn 47:352-359

Queney P (1947) Theory of perturbations in stratified currents with application to airflow over mountain barrier. The University of Chicago Press, Misc. Rep. No. 23, p 81

Queney P (1948) The problem of airflow over mountains: a summary of theoretical studies. Bull Am Meteorol Soc 29:16-26

Sarker RP (1965) A theoretical study of mountain waves on Western Ghats. Indian J Meteorol Geophys 16(4):565-584

Sarker RP (1966) A dynamical model of orographic rainfall. Mon. Wea. Rev. 94:555-572

Sarker RP (1967) Some modifications in a dynamical model of orographic rainfall. Mon Weather Rev 95:673-684

Sarker RP, Sinha Ray KC, De US (1978) Dynamics of orographic rainfall. Indian J Meteorol Geophys 29:335-348

Sawyar JS (1962) Gravity waves in the atmosphere as a 3-D problem. Quart J R Meteorol Soc 88:412-425

Sawyer JS (1960) Numerical calculation of the displacements of a stratified airstream crossing a ridge of small height. Quart J R Meteorol Soc 86:326-345

Scorer RS (1949) Theory of waves in the lee of mountain. Quart J R Meteorol Soc 45:41-56

Scorer RS, Wilkinson M (1956) Waves in the lee of an isolated hill. Quart J R Meteorol Soc 82:419-427

Sinha Ray KC (1988) Some studies on effects of Orography on airflow and rainfall. $\mathrm{PhD}$ thesis, University of Pune, India

Smith RB (1979) The influence of mountains on the atmosphere. Adv Geophys 21:87-230

Smith RB (1980) Linear theory of stratified flow past an isolated mountain. Tellus 32:348-364

Wurtele MG (1957) The three-dimensional lee wave. Beitr Phys Frei Atmos 29:242-252 